\title{
Hypertension artérielle pulmonaire, moelle osseuse, précurseurs endothéliaux et sérotonine
}

Estelle Ayme-Dietrich ${ }^{2}$, Sophie M. Banas ${ }^{1}$, Laurent $_{\text {Monassier }}^{2}$ et Luc Maroteaux ${ }^{1 *}$

${ }^{1}$ INSERM UMR-S 839, F75005, Paris, France; Université Pierre et Marie Curie, F75005, Paris, France; Institut du Fer à Moulin, 17 rue du Fer à Moulin F75005, Paris, France.

${ }^{2}$ Laboratoire de Neurobiologie et Pharmacologie Cardiovasculaire EA7296, Faculté de Médecine, Fédération de Médecine Translationnelle de Strasbourg, Université et Centre Hospitalier de Strasbourg, Strasbourg, France

*Auteur correspondant: Luc Maroteaux, luc.maroteaux@upmc.fr

Reçu le 18 mars 2016

Titre courant : Sérotonine et hypertension artérielle pulmonaire

Résumé - La sérotonine et les cellules souches dérivées de la moelle osseuse participent de concert à l'hypertension artérielle pulmonaire. Notre travail a montré que l'absence du récepteur 5- $\mathrm{HT}_{2 \mathrm{~B}}$ génère des altérations permanentes de la composition du sang et de la moelle osseuse dans les lignées myéloïdes et, en particulier, dans les cellules progénitrices endothéliales. Dans l'hypertension artérielle pulmonaire (HTAP), les récepteurs 5- $\mathrm{HT}_{2 \mathrm{~B}}$ portés par les cellules de la moelle osseuse jouent un rôle fondamental. Ils contribuent à la différenciation / prolifération / mobilisation des précurseurs endothéliaux de la moelle. Ces cellules dérivées de la moelle osseuse ont une fonction critique dans le développement de l'HTAP et du remodelage vasculaire pulmonaire. Ces données indiquent que les cellules de la moelle osseuse jouent un rôle clé dans la pathogenèse de l'HTAP et suggèrent que les interactions entre la sérotonine et BMPR2 (bone morphogenic protein type 2 receptor) pourraient se situer dans la moelle osseuse.

Mots-clés : Plaquettes sanguines / récepteurs de la sérotonine / précurseurs endothéliaux / cellules souches hématopoïétiques / hypertension artérielle pulmonaire.

\section{Abstract : Pulmonary arterial hypertension, bone marrow, endothelial cell precursors and serotonin.}

Serotonin and bone-marrow-derived stem cells participate together in triggering pulmonary hypertension. Our work has shown that the absence of 5- $\mathrm{HT}_{2 \mathrm{~B}}$ receptors generates permanent changes in the composition of the blood and bone-marrow in the myeloid lineages, particularly in endothelial cell progenitors. The initial functions of 5- $\mathrm{HT}_{2 \mathrm{~B}}$ receptors in pulmonary arterial hypertension (PAH) are restricted to bone-marrow cells. They contribute to the differentiation / proliferation / mobilization of endothelial progenitor cells from the bone-marrow. Those bone-marrow-derived cells have a critical role in the development of $\mathrm{PAH}$ and pulmonary vascular remodeling. These data indicate that bone-marrow derived endothelial progenitors play a key role in the pathogenesis of $\mathrm{PAH}$ and suggest that interactions involving serotonin and bone morphogenic protein type 2 receptor (BMPR2) could take place at the level of the bone-marrow. 
Keywords: Blood platelets / serotonin receptors / endothelial precursors / hematopoietic stem cells / pulmonary arterial hypertension.

\author{
Abréviations \\ 5-HT : sérotonine \\ BMPR2 : bone morphogenic protein type 2 receptor \\ HIF: Hypoxia inducible factor \\ HTAP : hypertension artérielle pulmonaire \\ ISRS : inhibiteurs sélectifs de la recapture de la sérotonine \\ SRC : proto-oncogène tyrosine-protéine kinase \\ SERT : transporteur de la sérotonine \\ Tph1 : tryptophane hydroxylase 1
}

\title{
Introduction
}

Dans un organisme adulte, la majeure partie de la sérotonine se trouve dans les plaquettes sanguines d'où elle est libérée lors des phénomènes d'activation et/ou d'agrégation, occasionnant des effets vasoconstricteurs et pro-agrégeants par rétrocontrôle positif. Le stock de sérotonine plaquettaire circulant provient des sites de synthèse principalement localisés dans la paroi intestinale, après chargement des plaquettes (Figure 1). En périphérie, ce médiateur contribue au contrôle de nombreuses fonctions cardiovasculaires et respiratoires puisqu'en plus de ses effets dans l'agrégation plaquettaire, il intervient dans la modulation de la pression artérielle systémique et pulmonaire, de la fréquence cardiaque et de la respiration. Il régule aussi des lits circulatoires particuliers comme les circulations cérébrale et coronaire. Finalement, on lui attribue un rôle physiopathologique majeur dans les tumeurs carcinoïdes, des tumeurs neuroendocrines développées à partir du tube digestif qui sécrètent d'importantes quantités de sérotonine (Guillet-Deniau et al., 1997).

Il peut sembler surprenant qu'un même médiateur puisse posséder autant de fonctions différentes. Cette diversité peut s'expliquer par le fait que la sérotonine agit par l'intermédiaire de nombreux récepteurs. Au cours des vingt dernières années, l'identification des récepteurs de la sérotonine a beaucoup progressé. Désormais, il apparaît qu'il y a 15 gènes codant pour des récepteurs différents, répartis en quatre sous-familles: 5- $\mathrm{HT}_{1 / 5}, 5-\mathrm{HT}_{2}, 5-\mathrm{HT}_{3}$, et 5- $\mathrm{HT}_{4 / 6 / 7}$ (Hoyer et al., 1994). Ces récepteurs sont regroupés en fonction de leur structure et de leur mode de couplage intracellulaire. Le récepteur 5- $\mathrm{HT}_{3}$ est le seul canal ionique de la famille alors que tous les autres sont classiquement couplés à des protéines G: Gi pour les $5-\mathrm{HT}_{1 / 5}$, Gs pour les 5- $\mathrm{HT}_{4 / 6 / 7}$ et finalement $\mathrm{Gq}$ pour les 5-HT . Des sous-types ont pu être distingués au sein de ces catégories. Ainsi, par exemple, il existe trois membres chez les récepteurs 5-HT $\left(5-\mathrm{HT}_{2 \mathrm{~A}, 2 \mathrm{~B}, 2 \mathrm{C}}\right)$.

\section{Sérotonine et poumons}

\subsection{Sérotonine et physiologie pulmonaire}

Il a été montré que le poumon est un émonctoire important pour la sérotonine. En effet, le poumon du chien anesthésié peut éliminer plus de $90 \%$ de la sérotonine injectée par voie intraveineuse (i.v.) en un "unique passage" (Thomas \& Vane, 1967). Les plaquettes sont connues pour recapter activement la sérotonine in vitro. En fait, la sérotonine sanguine, ayant 
pour origine les cellules entérochromaffines de la paroi intestinale, est stockée dans les granules denses des plaquettes. Chez la souris éveillée, une translocation rapide des plaquettes dans les poumons est induite par la stimulation des récepteurs 5-HT 2 . Les plaquettes s'accumulent dans les poumons dans les 6 secondes suivant une injection de sérotonine ou d'ADP, chez la souris (Yu et al., 2009). Ainsi, la translocation des plaquettes dans les poumons et la recapture de sérotonine par des plaquettes peuvent se produire presque immédiatement après l'injection de sérotonine. La stimulation du récepteur 5- $\mathrm{HT}_{2 \mathrm{~A}}$ (principal récepteur de la sérotonine dans les plaquettes) (De Clerck et al., 1984) serait responsable de ce phénomène de translocation des plaquettes dans les poumons en réponse à la sérotonine (Figure 1).

Il a été rapporté que 20 à $50 \%$ des mégacaryocytes matures finissent par atteindre le poumon chez l'homme, et qu'entre 7 et $17 \%$ des plaquettes sont libérées localement (ZuckerFranklin \& Philipp, 2000). Il apparaît de plus en plus clairement que les plaquettes sont libérées dans le sang à partir des mégacaryocytes lorsque des extensions des mégacaryocytes sortent au travers des sinusoïdes de la moelle ou lors d'un processus similaire existant dans les poumons, le premier lit capillaire rencontré par une cellule quittant la moelle osseuse. C'est dans les poumons que la sérotonine injectée par voie i.v. rencontre aussi les premiers capillaires. Ainsi, il est probable que cette sérotonine puisse activer les plaquettes déjà présentes dans les poumons, mais aussi recruter les plaquettes circulantes, ce qui conduirait à l'accumulation de plaquettes dans le parenchyme pulmonaire (Yu et al., 2009). Par conséquent, l'accumulation pulmonaire des plaquettes joue un rôle important dans leur charge en sérotonine et dans l'élimination pulmonaire de la sérotonine, au moins chez la souris, et peut représenter l'un des mécanismes de protection de l'organisme contre la sérotonine.

\subsection{Sérotonine et hypertension pulmonaire}

L'hypertension artérielle pulmonaire (HTAP) est une maladie progressive caractérisée par une dysfonction endothéliale et un remodelage vasculaire des artérioles pulmonaires. L'augmentation progressive de la pression artérielle pulmonaire provoque une surcharge du ventricule droit qui conduit à son dysfonctionnement, son hypertrophie, puis à sa défaillance. L'HTAP est le terme clinique classiquement utilisé pour décrire une pathologie dont les premiers symptômes apparaissent quand la pression artérielle pulmonaire systolique moyenne est supérieure à $30 \mathrm{~mm} \mathrm{Hg}$ (au repos). L'HTAP idiopathique est rare (prévalence 1-2 pour 1.000.000 individus) et le pronostic est sombre (survie de moins de 3 ans à partir du diagnostic, en absence de traitement). L'HTAP est morphologiquement et histologiquement caractérisée par une néomuscularisation des petites artères pulmonaires avec une intima épaisse, une hypertrophie de la média, une prolifération adventitielle, et un dépôt anormal de matrice extracellulaire. La progression du remodelage vasculaire entraîne un rétrécissement de la lumière des artérioles, une augmentation de la pression artérielle pulmonaire, et enfin une hypertension artérielle pulmonaire irréductible (Chan \& Loscalzo, 2008). En dehors de cette forme idiopathique, l'hypertension pulmonaire primitive peut être provoquée, à titre d'exemples, par une anémie hémolytique, une infection par le VIH, ou des mutations génétiques dans le gène $B M P R 2$. Un séjour prolongé en haute altitude peut, du fait de l'hypoxie chronique, aussi induire une hypertension pulmonaire chez les personnes prédisposées. En outre, la prise d'anorexigènes a été associée à l'augmentation de la prévalence de l'HTAP. Dans les années 1980 et 1990, l'utilisation prolongée d'anorexigènes comme la dexfenfluramine et la fenfluramine, maintenant retirées du marché, a augmenté le risque de développer une hypertension pulmonaire d'un facteur 23 (Louis, 1999). Plus récemment, une augmentation de la pression artérielle pulmonaire et des cas de maladies 
cardiaques valvulaires ont été observés chez les patients atteints de la maladie de Parkinson traités avec le pergolide ou la cabergoline.

De nombreux arguments sont à l'origine de l'intérêt actuel suscité par la sérotonine dans le développement de l'HTAP expérimentale et humaine. Des données expérimentales ont permis d'authentifier l'action déterminante de la sérotonine dans la physiopathologie de l'HTAP induite par l'hypoxie. Un taux élevé de sérotonine plasmatique est corrélé avec une hypertension pulmonaire primitive (Kéreveur et al., 2000). La sérotonine est connue pour son action vasoconstrictrice et mitotique sur les cellules musculaires lisses vasculaires. L'expression de SERT (transporteur de la sérotonine) et des récepteurs 5- $\mathrm{HT}_{1 \mathrm{~B}, 2 \mathrm{~A}, 2 \mathrm{~B}}$ et ${ }_{7}$ de la sérotonine est détectée dans les cellules endothéliales et musculaires lisses de l'artère pulmonaire et de ses branches. L'analyse de la réponse mitotique de cellules musculaires lisses isolées à partir d'artères pulmonaires normales, ou provenant de patients atteints d'HTAP, confirme l'activité mitotique possible de la sérotonine sur les cellules musculaires lisses. Les cellules musculaires lisses des patients atteints d'HTAP prolifèrent davantage en réponse à la sérotonine et au sérum que les cellules de sujets témoins (Liu et al., 2011).

Chez les rongeurs, l'hypoxie chronique provoque un important remodelage vasculaire. Le remodelage des vaisseaux et l'hypertension pulmonaire hypoxique sont réduits chez les souris invalidées pour les gènes codant pour le récepteur $5-\mathrm{HT}_{1 \mathrm{~B}}$, la tryptophane hydroxylase 1 (Tph1) (enzyme limitante de la synthèse de sérotonine en périphérie), ou pour SERT (Chan \& Loscalzo, 2008). Par conséquent, les voies de signalisation de la sérotonine modulent la fonction des cellules endothéliales et des muscles lisses vasculaires pulmonaires, à la fois au stade normal et au stade de la maladie, et contribuent probablement à la progression de l'HTAP. Les inhibiteurs sélectifs de la recapture de la sérotonine (ISRS), qui augmentent les concentrations extracellulaires de sérotonine, mais inhibent le transport de la sérotonine, n'ont aucun effet sur le développement de l'HTAP hypoxique. Au contraire, une association entre l'utilisation maternelle tardive des ISRS et une hypertension pulmonaire néonatale persistante a été observée (Chambers et al., 2006). Par conséquent, il n'est actuellement pas clair si la sérotonine elle-même ou l'association de ses effecteurs principaux conduisent au développement de l'HTAP.

Des concentrations plasmatiques élevées en sérotonine ont été retrouvées chez la plupart des patients atteints d'HTAP. Une corrélation positive entre ces concentrations et la pression ventriculaire droite a été observée chez plus de $80 \%$ des patients présentant une HTAP (Hervé et al., 1995). Les composés qui peuvent provoquer une hypertension pulmonaire, telles que le pergolide, le cabergolide, la fenfluramine, ou la dexfenfluramine (ou leurs métabolites actifs) sont des substances capables de provoquer la libération de sérotonine, mais sont surtout des agonistes des récepteurs $5-\mathrm{HT}_{2}$ et plus précisément du sous-type $2 \mathrm{~B}$ (5$\mathrm{HT}_{2 \mathrm{~B}}$ ). Cette stimulation de la libération de sérotonine pourrait contribuer à l'augmentation des concentrations plasmatiques de ce médiateur et favoriser l'activation des récepteurs sérotoninergiques. D'ailleurs, une exposition chronique à l'hypoxie (simulant une haute altitude) entraîne une surexpression des récepteurs 5- $\mathrm{HT}_{2 \mathrm{~B}}$ (et 5- $\mathrm{HT}_{1 \mathrm{~B}}$ ) chez les souris (comme chez les patients atteints d'HTAP) (Launay et al., 2002). De surcroît, l'HTAP induite par l'hypoxie chez les souris est significativement potentialisée par l'administration de doses thérapeutiques de dexfenfluramine. Au contraire, la survenue de l'HTAP induite par l'hypoxie chez les souris est complètement reversée en présence d'un antagoniste sélectif du récepteur $5-\mathrm{HT}_{2 \mathrm{~B}}$, le RS-127445. De manière similaire, les souris invalidées pour le gène du récepteur $5-\mathrm{HT}_{2 \mathrm{~B}}\left(\mathrm{Htr}_{2 \mathrm{~B}}{ }^{-1-}\right)$ ne développent pas d'HTAP à la suite d'une exposition chronique à l'hypoxie et/ou à l'administration de dexfenfluramine (Launay et al., 2002) (Figure 2).

Plus récemment, des adultes habitués à l'exercice en haute altitude ont été traités avec un composé antagoniste sélectif des récepteurs 5-HT ${ }_{2 \mathrm{~B}}$ développé par la firme Epix, le PRX08066. Ces individus sains ont montré une réduction de la pression systolique 
pulmonaire durant un exercice en hypoxie. Un essai clinique de Phase II étudiant les effets de ce composé dans l'hypertension artérielle pulmonaire secondaire à une broncho-pneumopathie chronique obstructive (BPCO) a été publié en août 2007. Sur 58 patients traités avec une dose de $400 \mathrm{mg}$, 44\% présentent une réduction de $4 \mathrm{~mm} \mathrm{Hg}$ de la pression systolique pulmonaire, le PRX-08066 n'ayant aucun effet sur la pression sanguine systémique (Rhodes et al., 2009). En utilisant la monocrotaline (MCT) comme modèle d'hypertension pulmonaire chez le rat, d'autres études ont confirmé que les antagonistes des récepteurs 5- $\mathrm{HT}_{2 \mathrm{~B}}$ (Terguride, PRX08066, ou C-122) réduisaient significativement les pressions pulmonaires, l'épaississement de la paroi et l'occlusion de la lumière, mais maintenaient les fonctions cardiaques (Porvasnik et al., 2010; Dumitrascu et al., 2011; Zopf et al., 2011).

\subsection{Sérotonine et BMPR2}

La sérotonine peut aussi influencer d'autres voies de régulation impliquées dans la progression de l'HTAP; en particulier, la sérotonine peut inhiber la voie de signalisation de BMP (bone morphogenic protein) par une modulation des protéines de signalisation en aval. Cette voie de signalisation est fortement suspectée dans la physiopathologie de l'HTAP car l'administration chronique de sérotonine augmente la pression artérielle sanguine et le remodelage vasculaire chez les souris hétérozygotes pour le gène $B m p r 2, B m p r 2^{+/}$, un effet potentialisé par l'hypoxie. Ces expériences mettent en évidence les interactions entre BMPR2 et la voie de signalisation de la sérotonine. Ces voies de signalisation et leur perturbation pourraient constituer d'importants cofacteurs de l'hypoxie dans la pathogenèse de l'HTAP (Long et al., 2006). Ceux-ci, en retour, peuvent moduler la voie de signalisation de BMPR2 par inhibition d'un de ses partenaires, BMPR1A. Si on intègre l'ensemble de ces données, les voies de la sérotonine et de BMP ne peuvent pas être les seuls facteurs pathogéniques, mais il semble légitime de penser que ces deux voies de signalisation empruntent des voies de régulation communes potentiellement impliquées dans cette maladie complexe. Ainsi des déterminants spécifiques génétiques, tels que des mutations de $B M P R 2$, pourraient interagir avec des perturbations environnementales comme l'hypoxie ou l'utilisation d'anorexigènes, et des gènes impliqués dans la pérennisation de la maladie tels que le récepteur $5-\mathrm{HT}_{2 \mathrm{~B}}$ ou SERT, pour mener à l'une des nombreuses expressions cellulaires, histologiques et cliniques de l'hypertension artérielle pulmonaire. Ainsi, il a été montré récemment que le blocage des récepteurs $5-\mathrm{HT}_{2 \mathrm{~B}}$ pouvait potentiellement empêcher l'apparition de l'HTAP familiale, dans la mesure où ce simple blocage inhibe la signalisation déclenchée par la phosphorylation de $S R C$ due à la mutation de Bmpr2 chez la souris (West et al., 2016).

\section{HTAP et cellules souches}

\subsection{HTAP et facteurs hématopoiétiques extrinsèques aux poumons}

Les niveaux plasmatiques de sérotonine sont augmentés chez les patients atteints d'HTAP, même après transplantation pulmonaire (Hervé et al., 1995), ce qui suggère que la sérotonine est un facteur causal extra-pulmonaire pour l'HTAP ou est associé à un tel facteur. La suppression de la tryptophane hydroxylase 1 (Tph1, enzyme limitante dans la synthèse de la sérotonine périphérique), du SERT, ou des récepteurs $5-\mathrm{HT}_{1 \mathrm{~B}}$ réduit le remodelage vasculaire pulmonaire et l'hypertension pulmonaire hypoxique (Morecroft et al., 2007). La mobilisation de cellules de la moelle osseuse dans la circulation sanguine est une réponse physiologique majeure secondaire à l'hypoxie. De manière intéressante, des cellules progénitrices de la moelle osseuse ont été localisées dans les poumons lors de l'HTAP, ce qui soulève la question 
de leur rôle dans la progression de la maladie. D'une part, des cellules souches, résidentes ou non, peuvent donner lieu à une proportion significative de cellules musculaires lisses qui contribuent à l'hyperplasie intimale et au remodelage vasculaire dans les poumons (Stenmark et al., 2006). Ces cellules progénitrices issues de la moelle osseuse peuvent également contribuer au remodelage de la paroi vasculaire qui est caractéristique de l'HTAP (Davie et al., 2004; Hayashida et al., 2005; Frid et al., 2006; Asosingh et al., 2008; Marsboom et al., 2008). On ne savait pas, cependant, si cette présence de progéniteurs représente une protection ou un processus d'aggravation du développement de l'HTAP (Diller et al., 2010). D'autres observations ont également identifié une association entre l'HTAP et des troubles hématologiques liés à la moelle osseuse (Yoder \& Rounds, 2011): dans les troubles de prolifération des cellules souches hématopoïétiques tels que les cancers myéloprolifératifs, il y a une incidence élevée et inexpliquée d'HTAP. L'HTAP est maintenant reconnue comme une complication des transplantations de moelle osseuse lors du traitement de certaines leucémies (Limsuwan et al., 2006), des troubles myéloprolifératifs chroniques (Farha et al., 2011), ou du traitement de l'ostéopétrose maligne infantile (Steward et al., 2004).

Les cellules c-kit ${ }^{+}$progénitrices dérivées de la moelle osseuse peuvent participer au remodelage vasculaire. L'infiltration de cellules c-kit ${ }^{+}$dans les lésions artérielles pulmonaires a été associée à une augmentation de l'expression de l'ARNm c-kit. Deux populations de cellules circulantes dérivée de la moelle (linCD $34^{\text {high }} \mathrm{CD} 133^{\text {high }}\left[\mathrm{c}^{-} \mathrm{kit}^{\text {high }} \mathrm{CXCR} 4^{\text {low }}\right]$ et lin $^{-}$ $\mathrm{CD} 34^{\text {low }} \mathrm{CD} 133^{-}$[c-kit $\left.^{\text {low }} \mathrm{CXCR} 4^{\text {high }}\right]$ ) sont augmentées dans l'HTAP par rapport aux sujets témoins. Les lésions artérielles pulmonaires sont associées à l'expression de CXCL12/SDF-1 $\alpha$ qui peut recruter les cellules c-kit ${ }^{+}$. Dans l'HTAP, les cellules c-kit ${ }^{+}$peuvent participer aux lésions artérielles pulmonaires et au remodelage vasculaire (Montani et al., 2011). L'HTAP est donc une vasculopathie caractérisée par une circulation importante de progéniteurs proangiogéniques $\mathrm{CD} 34^{+} \mathrm{CD} 133^{+}$. Le nombre de cellules progénitrices $\mathrm{CD} 34^{+} \mathrm{CD} 133^{+}$est ainsi plus élevé dans la moelle osseuse, le sang et les artères pulmonaires des patients présentant une HTAP que chez les témoins sains. Les facteurs myéloïdes HIF-inductibles, érythropoïétine, SCF et HGF sont également présents à des niveaux plus élevés dans le sang des patients atteints d'HTAP que chez les sujets sains (Farha et al., 2011). Les précurseurs circulants des cellules endothéliales $\mathrm{CD} 34^{+} \mathrm{CD} 133^{+} \mathrm{VEGF}^{+}$sont augmentés chez les patients atteints d'HTAP (Toshner \& Morrell, 2010). C'est la population à croissance tardive, $\mathrm{CD} 133^{+} \mathrm{c}-\mathrm{kit}^{+} \mathrm{CXCR} 4^{+}$, qui a été associée aux lésions plexiformes et qui montre un phénotype hyperprolifératif avec altération de la capacité à former des réseaux vasculaires, et ainsi pourrait contribuer au remodelage pathologique (Toshner et al., 2009). Nos études montrent enfin que le récepteur $5-\mathrm{HT}_{2 \mathrm{~B}}$ est exprimé sur les précurseurs circulants c-kit ${ }^{+}$. En outre, des cultures ex vivo de cellules progénitrices $\mathrm{CD} 34^{+}$humaines en présence d'un antagoniste des récepteurs de $5-\mathrm{HT}_{2 \mathrm{~B}}$ donnent lieu à une altération de la différenciation myéloïde, ce qui indique un rôle central de la sérotonine, par l'intermédiaire des récepteurs 5-HT $2 \mathrm{~B}$, dans la génération de précurseurs associés à l'HTAP (Launay et al., 2012). La sérotonine augmente l'expansion ex vivo de cellules $\mathrm{CD}_{3} 4^{+}$de la moelle humaine, en exerçant des effets antiapoptotiques par l'intermédiaire du récepteur 5-HT $2 \mathrm{~B}$ (Yang et al., 2007; Thomas et al., 2013).

\subsection{Sérotonine et hématopoiièse}

Récemment, il a été montré que l'absence de sérotonine réduisait les précurseurs érythroïdes dans la moelle osseuse par l'intermédiaire des récepteurs $5-\mathrm{HT}_{2 \mathrm{~A}}$ et $5-\mathrm{HT}_{2 \mathrm{~B}}$. En effet, le PNU22394, un agoniste des récepteurs $5-\mathrm{HT}_{2}$, produit le même effet prolifératif que la sérotonine sur les précurseurs érythroïdes (Amireault et al., 2011). Aux premiers stades de la mégacaryopoïèse, la sérotonine régule aussi la prolifération et la survie des mégacaryoblastes via des effets anti-apoptotiques (Liu \& Yang, 2006). Un prétraitement avec de la sérotonine 
protège aussi la lignée mégacaryocytaire HEL de l'apoptose induite par le NO (Battinelli \& Loscalzo, 2000). Nous avions précédemment montré que les récepteurs 5-HT $2 \mathrm{~B}$ sont nécessaires pour la prolifération et la survie des cardiomyocytes nouveau-nés via la régulation de la perméabilité membranaire mitochondriale, l'activation des caspases, et Akt/ERK1 (Nebigil et al., 2003). Indépendamment, la sérotonine stimule les cellules stromales de la moelle osseuse humaine et agit en synergie avec d'autres facteurs de croissance qui favorisent la mobilisation des cellules souches et progénitrices hématopoïétiques (Yang et al., 2007). La sérotonine augmente la mégacaryopoï̀se et la formation de proplaquettes via ERK1/2 et la réorganisation du cytosquelette via le récepteur 5- $\mathrm{HT}_{2 \mathrm{~B}}$ identifié dans les mégacaryocytes. La sérotonine aurait un effet puissant sur la formation des plaquettes et cet effet est probablement contrôlé par le récepteur 5- $\mathrm{HT}_{2 \mathrm{~B}}$ avec l'activation subséquente de $\mathrm{p}$-ERK1/2, la réorganisation de la F-actine et la formation des proplaquettes (Ye et al., 2014). Les souris n'exprimant pas de récepteurs 5- $\mathrm{HT}_{2 \mathrm{~B}}$ présentent des modifications dans le nombre de plaquettes, de monocytes-macrophages et dans les lignées myéloïdes de la moelle osseuse (Figure 3).

\subsection{Sérotonine, hématopoīèse et HTAP}

De manière intéressante, nous avons rapporté que des souris invalidées pour le gène du récepteur $5-\mathrm{HT}_{2 \mathrm{~B}}$ et transplantées avec de la moelle osseuse de souris contrôle, montrent après irradiation une augmentation de la pression pulmonaire et du remodelage vasculaire induite par l'hypoxie ou par l'injection de monocrotaline, alors que l'élimination des récepteurs 5$\mathrm{HT}_{2 \mathrm{~B}}$ limitée aux cellules de la moelle osseuse chez des souris témoins confère une résistance complète (Launay et al., 2012). Ces résultats montrent clairement que la contribution des récepteurs $5-\mathrm{HT}_{2 \mathrm{~B}}$ dans l'HTAP est extrinsèque au poumon et dérive de la moelle osseuse, indépendamment des cellules pulmonaires résidentes. Dans les lignées de cellules souches hématopoïétiques, la sérotonine module probablement l'équilibre entre les différentes lignées en participant à la prolifération et / ou la survie de la lignée spécifique via ses récepteurs. Cependant, l'action de la sérotonine sur l'hématopoïèse ou sur le microenvironnement de la moelle osseuse dans des conditions physiopathologiques reste à préciser.

La suite de notre travail visait à évaluer la contribution de cellules issues de la moelle dans l'HTAP en caractérisant les cellules souches circulantes CD34 ${ }^{+}$. Nous avons émis l'hypothèse selon laquelle ces cellules pouvaient être des cellules progénitrices endothéliales, d'origine médullaire, envahissant le tissu pulmonaire pathologique. La mobilisation de cellules souches de la moelle osseuse est une réponse physiologique à des conditions hypoxiques (Frid et al., 2006; Asosingh et al., 2008), et des précurseurs circulants dérivés de la moelle osseuse sont augmentés dans l'HTAP (Davie et al., 2004; Hayashida et al., 2005; Sata, 2006). Les patients atteints de syndromes myéloprolifératifs développent souvent une HTAP (Popat et al., 2006). Il a été démontré que les cellules de la lignée mononucléaire pouvaient se différencier en cellules endothéliales et en cellules musculaires lisses (Asahara et al., 1997; Shimizu et al., 2001; Bailey et al., 2006; Sata, 2006) et que des précurseurs circulants de cette lignée pouvaient contribuer au remodelage des vaisseaux pulmonaires induit par l'hypoxie (Frid et al., 2006). À ce stade, ce processus, semblant finalement contribuer à la prolifération anormale des cellules musculaires lisses et des cellules endothéliales et conduisant à la progression de l'hypertension pulmonaire, était encore hypothétique (Diller et al., 2010). Notre travail montre que l'absence du récepteur 5-HT $2 \mathrm{~B}$ génère des altérations permanentes de la composition du sang et de la moelle osseuse dans les lignées myéloïdes et, en particulier, dans les cellules progénitrices endothéliales. Le rôle des récepteurs $5-\mathrm{HT}_{2 \mathrm{~B}}$ dans la maturation des différents précurseurs myéloïdes, dont les 
précurseurs immatures des cellules endothéliales, requis pour la pathogenèse de l'HTAP, reste encore à décrypter.

En utilisant comme modèle génétique d'HTAP les souris mutantes Bmpr2, une étude récente a confirmé le rôle des cellules circulantes dérivées de la moelle osseuse dans l'initiation de cette pathogenèse. Des cellules de moelle osseuse exprimant une mutation dans le gène Bmpr2 (Bmpr2R899X) provoquent une HTAP avec remodelage et inflammation, lorsqu'elles sont transplantées dans des souris contrôles, alors que des cellules de moelle osseuse témoin ont un effet protecteur contre le développement de la maladie, lorsqu'elles sont transplantées dans des souris mutantes. L'analyse fonctionnelle et moléculaire identifie pour les cellules mutantes des dysfonctions suggestives d'un phénotype d'HTAP, peu après l'activation du transgène et bien avant le développement de la pathologie pulmonaire. Ces données étayent nos résultats indiquant que les cellules de la moelle osseuse jouent un rôle clé dans la pathogenèse de l'HTAP et suggèrent que les interactions sérotonine/BMPR2 pourraient se situer dans la moelle osseuse (Yan et al., 2015).

Nous avions montré précédemment que le traitement chronique de souris par la dexfenfluramine $(2,5 \mathrm{mg} / \mathrm{kg} / \mathrm{j})$ conduit à la conversion quantitative de ce composé en nordexfenfluramine, avec une concentration plasmatique de $500 \mathrm{nM}$ en fin de traitement dans ces conditions, et que ce métabolite est un agoniste sélectif des récepteurs $5-\mathrm{HT}_{2 \mathrm{~B}}$. Nous avons donc testé l'effet aigu de ce métabolite sur la mobilisation des cellules de la moelle. Une série d'expériences préliminaires indique que cette stimulation aiguë par la nordexfenfluramine, un agoniste des récepteurs $5-\mathrm{HT}_{2 \mathrm{~B}}$, provoque une augmentation rapide de précurseurs endothéliaux $\mathrm{CD} 34^{+} \mathrm{CD} 31^{+}$dans la moelle et dans le sang, indiquant que ce composé agit directement sur la mobilisation de ces précurseurs endothéliaux via l'activation des récepteurs $5-\mathrm{HT}_{2 \mathrm{~B}}$.

\section{Conclusion}

La sérotonine et les cellules souches dérivées de la moelle osseuse participent de concert à l'HTAP: (i) les fonctions initiales dans l'hypertension artérielle pulmonaire des récepteurs 5$\mathrm{HT}_{2 \mathrm{~B}}$ sont restreintes aux cellules de la moelle osseuse, (ii) les récepteurs 5- $\mathrm{HT}_{2 \mathrm{~B}}$ contribuent à la différenciation/prolifération/mobilisation des précurseurs endothéliaux de la moelle, et (iii) les cellules dérivées de la moelle osseuse ont une fonction critique dans le développement de l'HTAP. Le récepteur 5- $\mathrm{HT}_{2 \mathrm{~B}}$ est ainsi impliqué dans la mobilisation des cellules souches de la moelle osseuse qui participent à la pathogénèse de l'HTAP et au remodelage vasculaire pulmonaire.

La prise en charge médicale de l'HTAP reste incomplète et le pronostic de sa forme idiopathique est toujours sombre en dépit des nouvelles options thérapeutiques offertes depuis quelques années. De plus, de nouvelles approches pharmacologiques sont en cours d'essai (Archer et al., 2010). À l'heure actuelle, les thérapies disponibles sont essentiellement des vasodilatateurs, bien utiles dans l'amélioration des symptômes fonctionnels et de la qualité de vie, mais qui ne sont que modérément efficaces sur le pronostic vital. Nous avons démontré que l'expression des récepteurs $5-\mathrm{HT}_{2 \mathrm{~B}}$ restreinte aux cellules de la moelle osseuse est nécessaire et suffisante pour le développement de l'HTAP par l'intermédiaire d'une action sur la différenciation des cellules souches hématopoïétiques. Des thérapies visant à atténuer la vascularisation artérielle pulmonaire, en agissant directement sur l'origine hématopoïétique des défauts responsables du remodelage vasculaire pulmonaire, seraient donc envisageables.

\section{Remerciements:}

Ce travail a été financé par le Centre National de la Recherche Scientifique, l'Institut National de la Santé et de la Recherche Médicale, l'Université Pierre et Marie Curie, et par des 
subventions de la Fondation pour la Recherche sur le Cerveau, la Fondation de France, la Fondation pour la Recherche Médicale "Equipe FRM DEQ2014039529", et le Ministère de la Recherche (Agence Nationale pour la Recherche ANR-12-BSV1-0015-01).

\section{Légendes des figures}

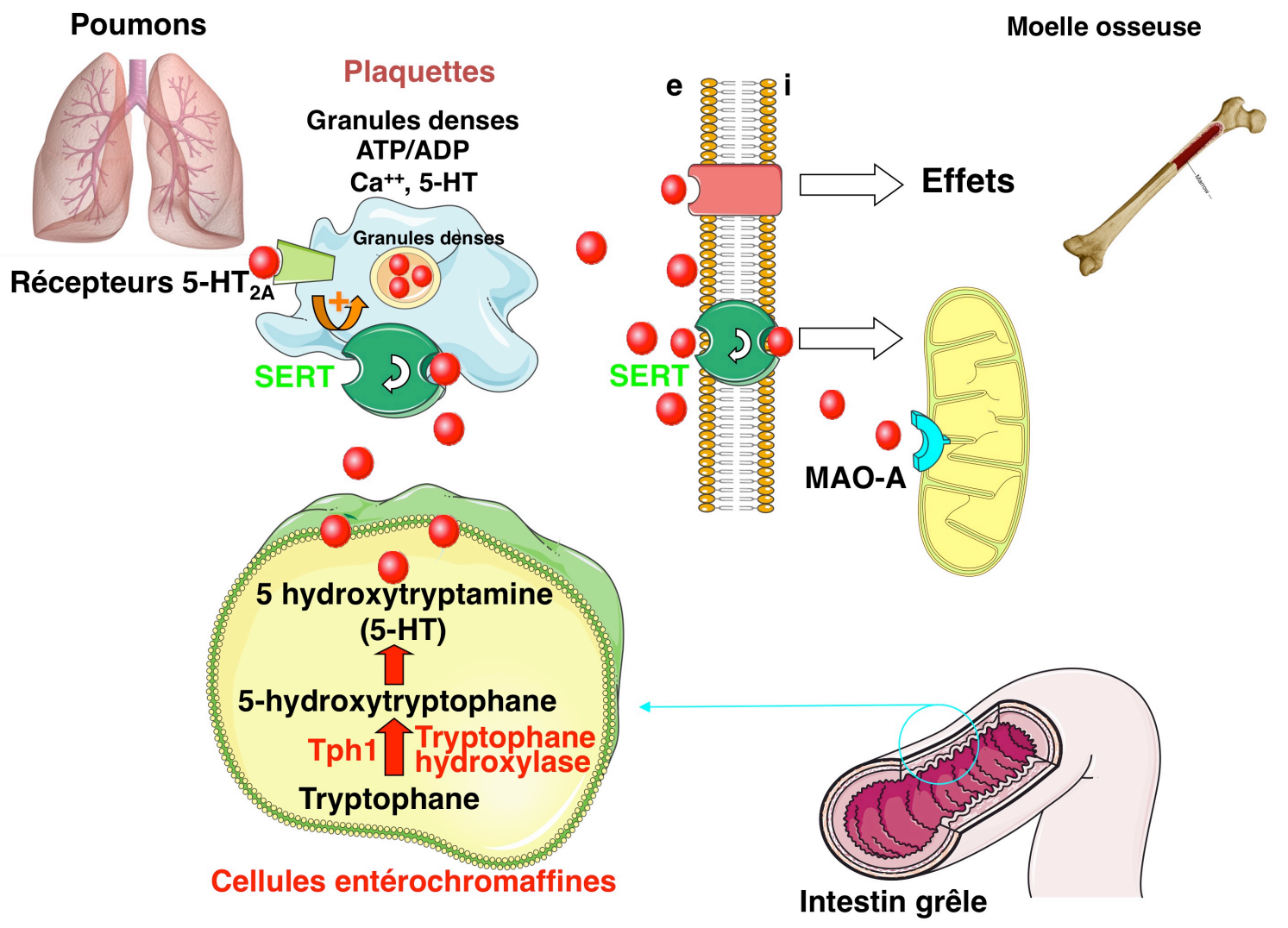

Figure 1 - La sérotonine périphérique- En périphérie, la sérotonine provient des sites de synthèse, principalement localisés dans la paroi intestinale, et est relarguée dans le système porte. Elle est chargée au niveau des poumons dans les plaquettes grâce au transporteur de la sérotonine (SERT) et est stockée dans les granules denses. La sérotonine est dégradée, dans les cellules qui expriment le transporteur, par la monoamine-oxydase A (MAO-A) localisée sur la paroi externe des mitochondries. La moelle osseuse est aussi une cible de la sérotonine périphérique. 


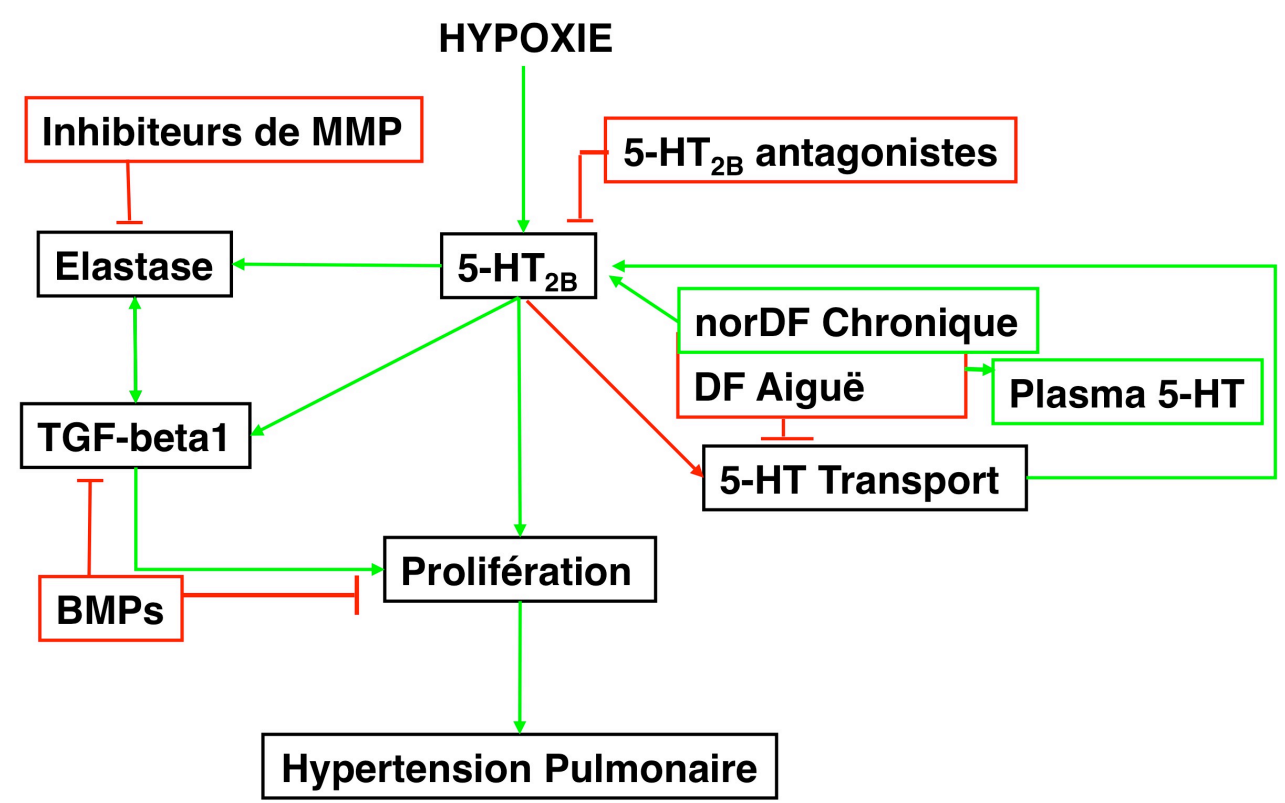

Figure 2 - L'activité du récepteur $5-\mathrm{HT}_{2 \mathrm{~B}}$ est nécessaire au développement de l'hypertension artérielle pulmonaire et régule les niveaux plasmatiques de sérotonine Chez la souris, l'hypoxie provoque une augmentation de la pression sanguine pulmonaire associée à un remodelage avec une prolifération de la paroi des artères pulmonaires, une augmentation de l'activité élastase et du niveau plasmatique de sérotonine et de TGFß1 (transforming growth factor-betal), ces paramètres étant potentialisés par le traitement de ces souris par la dexfenfluramine (DF) en injection aiguë ou son métabolite la nordexfenfluramine (norDF) lors d'une exposition chronique. Par contre, les souris présentant une invalidation soit génétique soit pharmacologique du récepteur 5- $\mathrm{HT}_{2 \mathrm{~B}}$ ne montrent aucun changement de ces paramètres. 


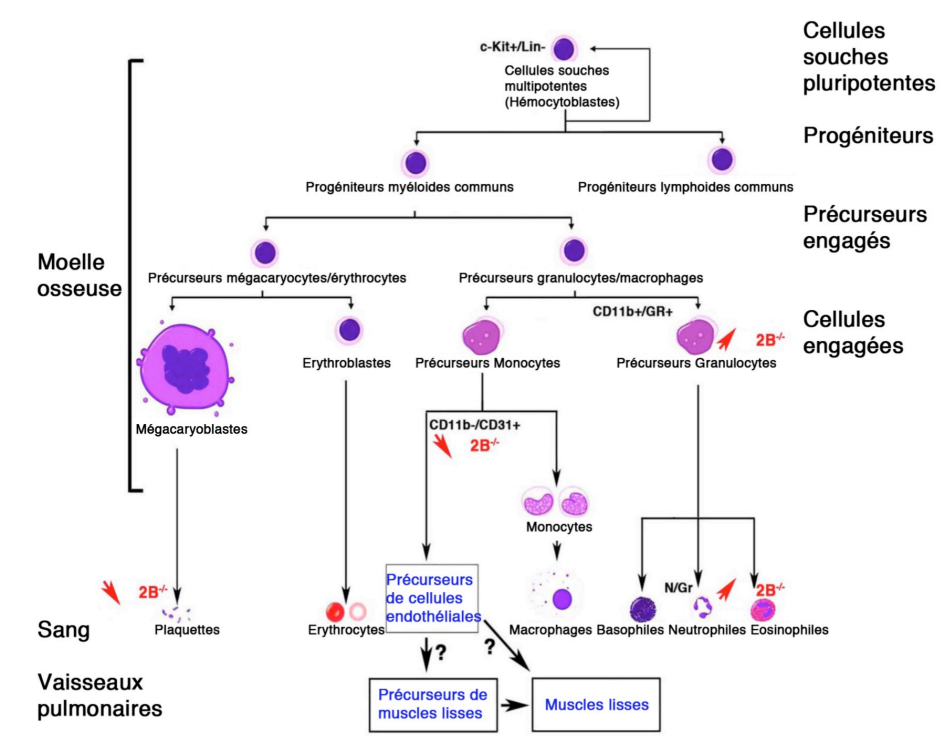

Figure 3 - Les récepteurs $5-\mathrm{HT}_{2 \mathrm{~B}}$ régulent la différenciation et la mobilisation de précurseurs hématopoiétiques - Les cellules souches hématopoïétiques impliquent la sérotonine et plusieurs récepteurs dont le récepteur 5- $\mathrm{HT}_{2 \mathrm{~B}}$ à différents niveaux de la lignée myéloïde. L'absence de récepteurs $5-\mathrm{HT}_{2 \mathrm{~B}}$ chez les souris mutantes $\left(\mathrm{Htr}_{2 B}{ }^{-1}, 2 \mathrm{~B}^{-1-}\right)$ conduit à une diminution $(\checkmark)$ des plaquettes et des progéniteurs endothéliaux immatures et de leur lignage, mais à une augmentation ( $(\nearrow)$ des précurseurs des granulocytes et de leur lignage.

\section{Références}

Amireault, P., Hatia, S., Bayard, E., Bernex, F., Collet, C., Callebert, J., Launay, J.-M., Hermine, O., Schneider, E., Mallet, J., Dy, M., and Côté, F. (2011). Ineffective erythropoiesis with reduced red blood cell survival in serotonin-deficient mice. Proc Natl Acad Sci USA, 108, 13141-13146.

Archer, S. L., Weir, E. K., and Wilkins, M. R. (2010). Basic Science of Pulmonary Arterial Hypertension for Clinicians: New Concepts and Experimental Therapies. Circulation, 121, 2045-2066.

Asahara, T., Murohara, T., Sullivan, A., Silver, M., van der Zee, R., Li, T., Witzenbichler, B., Schatteman, G., and Isner, J. M. (1997). Isolation of putative progenitor endothelial cells for angiogenesis. Science, 275, 964-967.

Asosingh, K., Aldred, M. A., Vasanji, A., Drazba, J., Sharp, J., Farver, C., Comhair, S. A. A., Xu, W., Licina, L., Huang, L., Anand-Apte, B., Yoder, M. C., Tuder, R. M., and Erzurum, S. C. (2008). Circulating angiogenic precursors in idiopathic pulmonary arterial hypertension. Am J Pathol, 172, 615-627. 
Bailey, A. S., Willenbring, H., Jiang, S., Anderson, D. A., Schroeder, D. A., Wong, M. H., Grompe, M., and Fleming, W. H. (2006). Myeloid lineage progenitors give rise to vascular endothelium. Proc Natl Acad Sci USA, 103, 13156-13161.

Battinelli, E., and Loscalzo, J. (2000). Nitric oxide induces apoptosis in megakaryocytic cell lines. Blood, 95, 3451-3459.

Chambers, C. D., Hernandez-Diaz, S., Van Marter, L. J., Werler, M. M., Louik, C., Jones, K. L., and Mitchell, A. A. (2006). Selective serotonin-reuptake inhibitors and risk of persistent pulmonary hypertension of the newborn. N Engl J Med, 354, 579-587.

Chan, S. Y., and Loscalzo, J. (2008). Pathogenic mechanisms of pulmonary arterial hypertension. J Mol Cell Cardiol, 44, 14-30.

Davie, N. J., Crossno, J. T., Frid, M. G., Hofmeister, S. E., Reeves, J. T., Hyde, D. M., Carpenter, T. C., Brunetti, J. A., McNiece, I. K., and Stenmark, K. R. (2004). Hypoxia-induced pulmonary artery adventitial remodeling and neovascularization: contribution of progenitor cells. Am J Physiol Lung Cell Mol Physiol, 286, L668-678.

De Clerck, F., Xhonneux, B., Leysen, J., and Janssen, P. A. (1984). Evidence for functional 5-HT2 receptor sites on human blood platelets. Biochem Pharmacol, 33, 2807-2811.

Diller, G.-P., Thum, T., Wilkins, M. R., and Wharton, J. (2010). Endothelial progenitor cells in pulmonary arterial hypertension. Trends Cardiovasc Med, 20, 22-29.

Dumitrascu, R., Kulcke, C., Königshoff, M., Kouri, F., Yang, X., Morrell, N., Ghofrani, H. A., Weissmann, N., Reiter, R., Seeger, W., Grimminger, F., Eickelberg, O., Schermuly, R. T., and Pullamsetti, S.S. (2011). Terguride ameliorates monocrotaline-induced pulmonary hypertension in rats. Eur Respir J, 37, 1104-1118.

Farha, S., Asosingh, K., Xu, W., Sharp, J., George, D., Comhair, S., Park, M., Tang, W. H. W., Loyd, J. E., Theil, K., Tubbs, R., Hsi, E., Lichtin, A., and Erzurum, S.C. (2011). Hypoxia-inducible factors in human pulmonary arterial hypertension: a link to the intrinsic myeloid abnormalities. Blood, 117, 3485-3493.

Frid, M. G., Brunetti, J.A., Burke, D.L., Carpenter, T.C., Davie, N.J., Reeves, J.T., Roedersheimer, M.T., van Rooijen, N., and Stenmark, K.R. (2006). Hypoxia-induced pulmonary vascular remodeling requires recruitment of circulating mesenchymal precursors of a monocyte/macrophage lineage. Am J Pathol, 168, 659-669.

Guillet-Deniau, I., Burnol, A.F., and Girard, J. (1997). Identification and localization of a skeletal muscle serotonin 5-HT2A receptor coupled to the Jak/STAT pathway. J Biol Chem, 272, 14825-14829.

Hayashida, K., Fujita, J., Miyake, Y., Kawada, H., Ando, K., Ogawa, S., and Fukuda, K. (2005). Bone marrow-derived cells contribute to pulmonary vascular remodeling in hypoxia-induced pulmonary hypertension. Chest, 127, 1793-1798.

Hervé, P., Launay, J.M., Scrobohaci, M.L., Brenot, F., Simonneau, G., Petitpretz, P., Poubeau, P., Cerrina, J., Duroux, P., and Drouet, L. (1995). Increased plasma serotonin in primary pulmonary hypertension. Am J Med, 99, 249-254.

Hoyer, D., Fozard, J.R., Saxena, P.R., Mylecharane, E.J., Clarke, D.E., Martin, G.R., and Humphrey, P.P.A. (1994). IUPHAR classification of receptors for 5-Hydroxytryptamine (serotonin). Pharmacol Rev, 46, 157-203.

Kéreveur, A., Callebert, J., Humbert, M., Hervé, P., Simonneau, G., Launay, J. M., \& Drouet, L. (2000). High plasma serotonin levels in primary pulmonary hypertension : effect of long-term epoprostenol (Prostacyclin) therapy. Arterioscler Thromb Vasc Biol, 20, 2233-2239.

Launay, J. M., Hervé, P., Peoc'h, K., Tournois, C., Callebert, J., Nebigil, C., Etienne, N., Drouet, L., Humbert, M., Simonneau, G., and Maroteaux, L. (2002). Function of the serotonin 5-hydroxytryptamine 2B receptor in pulmonary hypertension. Nat Med, 8, 1129-1135. 
Launay, J.-M., Hervé, P., Callebert, J., Mallat, Z., Collet, C., Doly, S., Belmer, A., Diaz, S. L., Hatia, S., Côté, F., Humbert, M., and Maroteaux, L. (2012). Serotonin 5-HT2B receptors are required for bone-marrow contribution to pulmonary arterial hypertension. Blood, 119, 1772-1780.

Limsuwan, A., Pakakasama, S., Rochanawutanon, M., and Hong-Eng, S. (2006). Pulmonary arterial hypertension after childhood cancer therapy and bone marrow transplantation. Cardiology, 105, 188-194.

Liu, Y. S., and Yang, M. (2006). The effect of 5-hydroxtryptamine on the regulation of megakaryocytopoiesis. Hematology, 11, 53-56.

Liu, Y., Wei, L., Laskin, D. L., and Fanburg, B. L. (2011). Role of protein transamidation in serotonin-induced proliferation and migration of pulmonary artery smooth muscle cells. Am J Resp Cell Mol Biol, 44, 548-555.

Long, L., MacLean, M. R., Jeffery, T.K., Morecroft, I., Yang, X., Rudarakanchana, N., Southwood, M., James, V., Trembath, R.C., and Morrell, N.W. (2006). Serotonin increases susceptibility to pulmonary hypertension in BMPR2-deficient mice. Circ Res, 98, 818-827.

Louis, W. J. (1999). Primary pulmonary hypertension and anorectic drugs. N Engl J Med, 340, 480-482.

Marsboom, G., Pokreisz, P., Gheysens, O., Vermeersch, P., Gillijns, H., Pellens, M., Liu, X., Collen, D., and Janssens, S. (2008). Sustained endothelial progenitor cell dysfunction after chronic hypoxia-induced pulmonary hypertension. Stem Cells, 26, 1017-1026.

Montani, D., Perros, F., Gambaryan, N., Girerd, B., Dorfmuller, P., Price, L. C., Huertas, A., Hammad, H., Lambrecht, B., Simonneau, G., Launay, J.-M., Cohen-Kaminsky, S., and Humbert, M. (2011). C-kit-positive cells accumulate in remodeled vessels of idiopathic pulmonary arterial hypertension. Am J Resp Crit Care Med, 184, 116-123.

Morecroft, I., Dempsie, Y., Bader, M., Walther, D. J., Kotnik, K., Loughlin, L., Nilsen, M., and MacLean, M. R. (2007). Effect of tryptophan hydroxylase 1 deficiency on the development of hypoxia-induced pulmonary hypertension. Hypertension, 49, 232-236.

Nebigil, C. G., Etienne, N., Messaddeq, N., and Maroteaux, L. (2003). Serotonin is a novel survival factor of cardiomyocytes: mitochondria as a target of 5-HT2B-receptor signaling. FASEB J, 17, 1373-1375.

Popat, U., Frost, A., Liu, E., Guan, Y., Durette, A., Reddy, V., and Prchal, J. T. (2006). High levels of circulating CD34 cells, dacrocytes, clonal hematopoiesis, and JAK2 mutation differentiate myelofibrosis with myeloid metaplasia from secondary myelofibrosis associated with pulmonary hypertension. Blood, 107, 3486-3488.

Porvasnik, S.L., Germain, S., Embury, J., Gannon, K.S., Jacques, V., Murray, J., Byrne, B.J., Shacham, S., and Al-Mousily, F. (2010). PRX-08066, a novel 5-hydroxytryptamine receptor $2 \mathrm{~B}$ antagonist, reduces monocrotaline-induced pulmonary arterial hypertension and right ventricular hypertrophy in rats. J Pharmacol Exp Ther, 334, 364-372.

Rhodes, C.J., Davidson, A., Gibbs, J.S., Wharton, J., and Wilkins, M. R. (2009). Therapeutic targets in pulmonary arterial hypertension. Pharmacol Ther, 121, 69-88.

Sata, M. (2006). Role of circulating vascular progenitors in angiogenesis, vascular healing, and pulmonary hypertension: lessons from animal models. Arterioscler Thromb Vasc Biol, 26, 1008-1014.

Shimizu, K., Sugiyama, S., Aikawa, M., Fukumoto, Y., Rabkin, E., Libby, P., and Mitchell, R. N. (2001). Host bone-marrow cells are a source of donor intimal smooth- muscle-like cells in murine aortic transplant arteriopathy. Nat Med, 7, 738-741.

Stenmark, K. R., Fagan, K. A., and Frid, M. G. (2006). Hypoxia-induced pulmonary vascular remodeling: cellular and molecular mechanisms. Circ Res, 99, 675-691. 
Steward, C.G., Pellier, I., Mahajan, A., Ashworth, M.T., Stuart, A.G., Fasth, A., Lang, D., Fischer, A., Friedrich, W., and Schulz, A.S. (2004). Severe pulmonary hypertension: a frequent complication of stem cell transplantation for malignant infantile osteopetrosis. Br J Haematol, 124, 63-71.

Thomas, D.P., and Vane, J.R. (1967). 5-hydroxytryptamine in the circulation of the dog. Nature, 216, 335-338.

Thomas, M., Ciuclan, L., Hussey, M.J., and Press, N.J. (2013). Targeting the serotonin pathway for the treatment of pulmonary arterial hypertension. Pharm Ther, 138, 409417.

Toshner, M., and Morrell, N.W. (2010). Endothelial progenitor cells in pulmonary hypertension - dawn of cell-based therapy? Int J Clin Practice Supp, 64, 7-12.

Toshner, M., Voswinckel, R., Southwood, M., Al-Lamki, R., Howard, L.S.G., Marchesan, D., Yang, J., Suntharalingam, J., Soon, E., Exley, A., Stewart, S., Hecker, M., Zhu, Z., Gehling, U., Seeger, W., Pepke-Zaba, J., and Morrell, N. W. (2009). Evidence of dysfunction of endothelial progenitors in pulmonary arterial hypertension. Am J Resp Crit Care Med, 180, 780-787.

West, J.D., Carrier, E.J., Bloodworth, N.C., Schroer, A.K., Chen, P., Ryzhova, L.M., Gladson, S., Shay, S., Hutcheson, J.D., and Merryman, W.D. (2016). Serotonin 2B Receptor Antagonism Prevents Heritable Pulmonary Arterial Hypertension. PloS One, 11, $\mathrm{e} 0148657$.

Yan, L., Chen, X., Talati, M., Nunley, B.W., Gladson, S., Blackwell, T., Cogan, J., Austin, E., Wheeler, F., Loyd, J., West, J., and Hamid, R. (2015). Bone Marrow-derived Cells Contribute to Pathogenesis of Pulmonary Arterial Hypertension. Am J Resp Cri Care Med, sous presse.

Yang, M., Li, K., Ng, P.C., Chuen, C.K., Lau, T.K., Cheng, Y.S., Liu, Y.S., Li, C.K., Yuen, P.M., James, A.E., Lee, S.M., and Fok, T.F. (2007). Promoting effects of serotonin on hematopoiesis: ex vivo expansion of cord blood CD34+ stem/progenitor cells, proliferation of bone marrow stromal cells, and antiapoptosis. Stem Cells, 25, 18001806.

Ye, J.Y., Liang, E.Y., Cheng, Y.S., Chan, G.C. F., Ding, Y., Meng, F., Ng, M.H.L., Chong, B.H., Lian, Q., and Yang, M. (2014). Serotonin Enhances Megakaryopoiesis and Proplatelet Formation via p-Erk1/2 and F-Actin Reorganization. Stem Cells, 32, $2973-$ 2982.

Yoder, M., and Rounds, S. (2011). Bad blood, bad endothelium: ill fate? Blood, 117, 34793480 .

Yu, Z., Ohba, M., Nakamura, M., Sasano, T., Ono, M., Sugawara, S., and Endo, Y. (2009). Dynamics of platelet mobilisation into lungs in response to 5-hydroxytryptamine (serotonin) in mice. Thromb Haemost, 102, 1251-1258.

Zopf, D.A., das Neves, L.A.A., Nikula, K.J., Huang, J., Senese, P.B., and Gralinski, M.R. (2011). C-122, a novel antagonist of serotonin receptor 5-HT(2B), prevents monocrotaline-induced pulmonary arterial hypertension in rats. Eur J Pharmacol, 670, 195-203.

Zucker-Franklin, D., and Philipp, C.S. (2000). Platelet production in the pulmonary capillary bed: new ultrastructural evidence for an old concept. Am J Pathol, 157, 69-74. 